EOMmun Communication et organisation

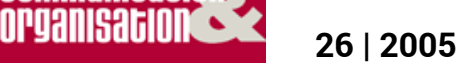

La communication des nouvelles éthiques de

l'entreprise

La muséologie. Histoire, développements, enjeux actuels

De André Gob et Noémie Drouguet, Paris, Armand Colin, 2003, 239 p., $21 €$

Isabelle Cousserand

C OpenEdition

Journals

Édition électronique

URL : http://journals.openedition.org/communicationorganisation/3304

DOI : 10.4000/communicationorganisation.3304

ISSN : $1775-3546$

Éditeur

Presses universitaires de Bordeaux

Édition imprimée

Date de publication : 2 janvier 2005

Pagination : 261-263

ISSN : 1168-5549

Référence électronique

Isabelle Cousserand, «La muséologie. Histoire, développements, enjeux actuels », Communication et organisation [En ligne], 26 | 2005, mis en ligne le 19 juin 2012, consulté le 21 septembre 2020. URL: http://journals.openedition.org/communicationorganisation/3304; DOI : https://doi.org/10.4000/ communicationorganisation.3304

Ce document a été généré automatiquement le 21 septembre 2020.

(c) Presses universitaires de Bordeaux 


\section{La muséologie. Histoire, développements, enjeux actuels}

De André Gob et Noémie Drouguet, Paris, Armand Colin, 2003, 239 p., $21 €$ Isabelle Cousserand

1 Cet ouvrage récent se présente comme une introduction à la science des musées, la muséologie. Selon les auteurs ${ }^{1}$, cette science "englobe tous les types et toutes les formes de musées et tous les aspects sous lesquels le musée peut être perçu $»^{2}$.

2 La muséologie en France connaît un renouveau autour des années 1970-1980 dans le sillage des actions et réflexions de Georges Henri Rivière (1897-1985) ${ }^{3}$, ancien directeur et conseiller permanent du Conseil international des musées (Icom).

3 C'est à compter des années 1980-1990, que le musée devient également et plus spécifiquement un objet d'étude en sciences de l'information et de la communication. Il est alors interrogé comme media, institution culturelle, lieu de médiation, territoire ou encore temple spectaculaire; on s'intéresse aussi à ses publics ou à l'usage des nouvelles technologies ${ }^{4}$. Même si le propos de l'ouvrage dépasse le cadre de la communication, les auteurs situent plus particulièrement la muséologie au croisement de quatre domaines scientifiques complémentaires: la sociologie, la pédagogie, la communication et bien entendu l'histoire.

4 Neuf chapitres servent un objectif large : introduire aux différents aspects que recouvre la muséologie. Il ne s'agit donc pas d'approfondir des questions très spécifiques mais de déployer l'éventail de la muséologie aujourd'hui. L'un des nombreux intérêts du travail proposé est qu'il ne cantonne évidemment pas à un genre de musée ou à sa fonction dite patrimoniale. Sa dynamique de traitement très transversale permet d'inclure de nombreuses formes muséales, quels que soient leur statut, leur objet, leur public et leur rayonnement.

5 Les auteurs présentent leur ouvrage en trois grandes parties. Après avoir défini en introduction la muséologie et rappelé les origines des musées et leur développement, la première partie, composée par les trois premiers chapitres, propose de définir et de délimiter le champ muséal (diversité des musées, musée et patrimoine immobilier). 
Puis elle s'intéresse aux rôles et fonctions du musée (les quatre fonctions, les attentes des utilisateurs, la conception, musée et identité) pour conclure sur les visiteurs (importance, fréquentation, connaissance et accueil du public, la démocratisation, le public-acteur dont les amis du musée).

6 La seconde partie regroupe les quatrième, cinquième, sixième et septième chapitres. Chacun aborde quatre fonctions dévolues au musée. La fonction de présentation à travers l'exposition s'apparente à un outil ou un vecteur de communication qui donne sens au musée (la structure de l'exposition, les objets, les textes, les reconstitutions, la scénographie, le multimédia, les visites guidées et les audioguides, l'évaluation). La fonction de conservation inscrit de fait le musée dans une perspective patrimoniale (le musée acteur du patrimoine, les acquisitions, les conditions de conservation et la gestion des risques, nettoyage et restauration, gardiennage et sécurité, protection contre les risques). La fonction scientifique renvoie à la recherche, au cœur des activités du musée (le catalogue, l'accès des chercheurs aux collections, les recherches sur le terrain, les publications, la recherche en muséologie). Enfin la fonction d'animation, que d'autres pourraient nommer médiation, positionne le musée en tant qu'acteur culturel inscrit dans un territoire plus ou moins étendu (l'insertion du musée dans la vie culturelle et sociale, les expositions temporaires, la visite guidée, le théâtre au musée, les animations pédagogiques, les activités culturelles, les services annexes).

7 La troisième et dernière partie caractérise le musée comme institution, à travers les dimensions architecturales et gestionnaires. Le huitième chapitre traite ainsi des spécificités architecturales des musées (salles, galeries, choix des matériaux, flexibilité des espaces, contraintes liées à la conservation, rénovation et réaffectation, fonctionnement du bâtiment). Le neuvième chapitre est consacré à la diversité de statut des musées, à leur financement et au personnel qu'ils emploient.

$8 \mathrm{Au}$ fil des chapitres, des illustrations ${ }^{5}$, quelques schémas et de nombreux textes encadrés viennent s'articuler autour du texte principal.

9 "Il n'y a pas de musée sans désir. Désir de séduire pour le concepteur de l'exposition, et désir d'être séduit pour le visiteur $"^{6}$, concluent les auteurs. Ainsi le musée nous conduit vers un ailleurs, à la lisière de nos fantasmes, là où quelquefois surgit le dépit de la frustration ou éclôt le plaisir du contentement.

10 Comme le sont les bons ouvrages dédiés à la connaissance générale d'un champ professionnel et scientifique particulier, celui-ci sert son lecteur, que ce dernier soit, ou non, néophyte. Sa construction est didactique, sa présentation fonctionnelle et le traitement du sujet exhaustif. Il suffit de jeter un œil sur la sélection de références qui est proposée en bibliographie pour en être convaincu. L'extrême soin apporté à son organisation raisonnée permet de brosser de manière méthodique différents sousthèmes du champ exploré et par la même occasion les différentes parties de l'ouvrage : ouvrages généraux et manuels, histoire des musées, muséologies spécialisées, définition - typologie et fonctions des musées, public, l'exposition, évaluation, conservation, animation - éducation - action culturelle, nouvelles technologies, formation en muséologie, architecture des musées, gestion, répertoires de musées, publications périodiques. L'ensemble est tout à fait complémentaire avec les notes de bas de page. S'il peut manquer une table des schémas et des illustrations, un index fourni vient renforcer l'impression d'ensemble : une introduction réussie à la muséologie. Du beau travail et du bel ouvrage. 


\section{NOTES}

1. Formés en histoire de l'art et archéologie, ils sont enseignants en muséologie à l'université de Liège.

2. P. 14.

3. Cf. l'ouvrage La muséologie selon Georges Henri Rivière, Paris, Dunod, 1989, 402 p. Etabli par d'anciens étudiants à partir de leurs notes de cours, il est enrichi de textes d'auteurs, proches de l'héritage légué par G.H. Rivière.

4. A titre d'exemples, on peut citer quelques ouvrages ou quelques contributions...

Eliséo Veron, Martine Levasseur, Ethnographie de l'exposition. L'espace, le corps et le sens, Centre Georges Pompidou, 1983. Jean Davallon, «le musée est-il vraiment un media? ", dans Publics et musées, $\mathrm{n}^{\circ} 2$ 2, 1992. Bernard Schiele, "L'invention simultanée du visiteur et de l'exposition ", dans Publics et musées, $n^{\circ} 2$, 1992. Paul Rasse (avec la collaboration d'Eric Necker), Techniques et cultures au musée. Enjeux, ingénierie et communication des musées de société, Lyon, PUL, 1997. J. Davallon, L'exposition à l'œuvre. Stratégie de communication et médiation symbolique, L'Harmattan, 1999. P. Rasse, Les musées à la lumière de l'espace public. Histoire, évolution, enjeux, L'Harmattan, 1999. François Mairesse, Le musée. Temple spectaculaire, Lyon, PUL, 2003.

5. Ce sont des photos noir et blanc qui présentent des aspects singuliers de divers musées européens, parfois peu connus.

6. P. 216. 